methods of cell reprograming than those used in this study.

Original article Dimos JT et al. (2008) Induced pluripotent stem cells generated from patients with ALS can be differentiated into motor neurons. Science 321: 1218-1221

\section{AMPA receptor endocytosis is a potential target in heroin addiction}

Inhibition of AMPA receptor endocytosis could help treat addiction to heroin. Van den Oever et al. have used a rat model to demonstrate that the link between re-exposure to preconditioned stimuli and heroin relapse could be explained by endocytosis of the AMPA receptor subunit GluR2, which leads in turn to synaptic depression in the medial prefrontal cortex.

The investigators re-exposed rats to cues that the animals had previously been trained to associate with heroin self-administration. Directly after re-exposure to these cues, AMPA receptor subunits GluR2 and GluR3 and NMDA receptor subtype $2 \mathrm{~B}$ were downregulated in synaptic membranes of the medial prefrontal cortex, and clathrin-coat assembly protein Ap2m1 was upregulated, as was shown by quantitative proteomics. Re-exposure of rats to heroin-conditioned cues also led to a corresponding reduction in AMPA currents, but not NMDA currents, in pyramidal neurons of the medial prefrontal cortex. Interestingly, rats trained to administer heroin, but that were not re-exposed to cues, had equal AMPA:NMDA current ratios to rats trained to self-administer saline, showing that heroin self-administration in the absence of heroin-conditioned cues does not trigger this mechanism. The cue-induced relapse seems to be dependent on clathrin-mediated AMPA receptor endocytosis in the ventral part of the medial prefrontal cortex, because cue-induced heroin-seeking decreased substantially when clathrin-dependent GluR2 endocytosis was blocked by a mimetic peptide of the GluR2 cytoplasmic tail. Similarly, this peptide was able to block cue-induced synaptic depression.

Original article Van den Oever MC et al. (2008) Prefrontal cortex AMPA receptor plasticity is crucial for cue-induced relapse to heroin-seeking. Nat Neurosci [doi:10.1038/nn.2165]

\section{Amyloid load has a role in dementia with Lewy bodies}

In a new study, dementia with Lewy bodies (DLB) has been associated with the deposition of amyloid- $\beta$ in the brain. According to Edison and colleagues, these results indicate that most patients with DLB could benefit from therapy with anti-amyloid agents.

The PET radiotracer ${ }^{11} \mathrm{C}$-labeled Pittsburgh Compound B ( $\left.{ }^{11} \mathrm{C}-\mathrm{PIB}\right)$ binds to amyloid- $\beta$ deposits in the cortex and striatum and is used to measure in vivo amyloid load in patients with Alzheimer disease. Edison et al. have now used ${ }^{11} \mathrm{C}$-PIB PET to assess amyloid load in patients with DLB or Parkinson disease dementia (PDD; $n=13$ and $n=12$, respectively), comparing findings with those from 10 patients with Parkinson disease without dementia and 41 age-matched healthy controls. Of the 13 patients with DLB, 11 (85\%) had a significantly increased amyloid load (indicated by increased ${ }^{11} \mathrm{C}$-PIB uptake) in at least one cortical region in comparison with that observed in the control group. By comparison, only $2(15 \%)$ patients with PDD demonstrated increased uptake of ${ }^{11} \mathrm{C}-\mathrm{PIB}$, and none of the patients with Parkinson disease without dementia showed an increase in uptake of the marker compared with controls.

The authors conclude that assessment of amyloid load can be used to differentiate between PDD and DLB and that ${ }^{11} \mathrm{C}$-PIB PET can help to target novel anti-amyloid agents to patients with DLB. Some patients with PDD might also benefit from such strategies.

Original article Edison P et al. (2008) Amyloid load in Parkinson's disease dementia and Lewy body dementia measured with [11C]PIB-PET. J Neurol Neurosurg Psychiatry [doi:10.1136/jnnp.2007.127878] 